\title{
A Study of Non-English Majors Postgraduates Students' Listening Anxiety and Its Countermeasures
}

\author{
Xiaoyuan Deng, Bingrong Li \\ North University of China, Taiyuan, China \\ Email: 1712886355@qq.com
}

How to cite this paper: Deng, X.Y. and Li, B.R. (2021) A Study of Non-English Majors Postgraduates Students' Listening Anxiety and Its Counter- measures. Open Access Library Journal, 8: e7963.

https://doi.org/10.4236/oalib.1107963

Received: September 14, 2021

Accepted: September 20, 2021

Published: September 23, 2021

Copyright $\odot 2021$ by author(s) and Open Access Library Inc.

This work is licensed under the Creative Commons Attribution International License (CC BY 4.0).

http://creativecommons.org/licenses/by/4.0/

\section{(c) (i) Open Access}

\begin{abstract}
Language anxiety is one of the important emotional factors affecting learning effect, especially in listening activities. Listening anxiety is common among English learners and can affect their academic performance. Rational use of listening learning strategies can reduce the negative impact of anxiety on English learning. This paper conducts a qualitative research on the status and causes of non-English major postgraduates' listening anxiety through interviews, and puts forward targeted countermeasures to overcome the listening anxiety.
\end{abstract}

\section{Subject Areas \\ Linguistics}

\section{Keywords}

English Listening, Anxiety, Strategies

\section{1. 引言}

自二十世纪九十年代以来, 越来越多研究者进行了提高语言学习效果的 研究。众多的教师和研究者对学习者情感因素给予更多的关注。外语焦虑作 为一种重要的情感因素, 是语言学习中所特有的一种复杂的情感现象。张健、 周莉薇[1]的研究表明, 非英语专业大学生普遍存在较高的听力焦虑, 而特殊 的听力学习活动可以降低听力焦虑。我们可以针对学生和教师在听力过程中 的不同角色提供相应的解决方案, 以缓解非英语专业研究生的英语听力焦虑。 因此，本文采取了访谈的形式，进行了定性的分析，探究导致听力焦虑的因 素有哪些以及应对策略, 以期提高学生的听力水平, 从而为听力教学注入新 
活力。

\section{2. 外语学习焦虑的研究背景}

外语焦虑是由于外语学习过程的特殊性, 学习者在外语课堂学习过程中 形成的与外语课堂学习相关的自我感知、信念、情绪和行为的综合体 [2]。听 力焦虑产生于外语学习者听力的理解过程中, 需要用目标语进行表达时, 学 习者会容易产生畏惧或担心的心理[3]。Krashen [4]提出了情感过滤假说, 认 为焦虑对语言习得有重大的负面影响。听力理解是一个复杂且较为困难的过 程, 在这个过程中, 学习者产生焦虑情绪在所难免, 然而焦虑情绪会让学生 面对听力内容的抓取时变得困难，从而使自身的听力理解能力无法进一步提 升, 所以解决学习者听力焦虑十分关键 [5]。虽然 Horwitz 等学者表示, 听力 是五大基本语言技能中最易使外语学习者产生焦虑的技能之一，但关于外语 听力焦虑的研究并不多, 可以说仍然处在起步阶段 [6]。

\section{3. 研究设计}

\section{1. 研究问题}

1) 非英语专业硕士研究生的外语课堂焦虑和听力焦虑状况及其主要影 响因素是什么?

2) 听力焦虑的主要来源是什么?

3）降低非英语专业研究生听力焦虑的对应策略有哪些?

\section{2. 研究对象}

英语听力在研究生阶段占有重要地位, 本研究随机选取某高校六位有高 听力焦虑的非英语专业研究生作为受试对象, 受调查者均有 14 年的英语学 龄。

\section{3. 研究工具}

本研究的研究工具是访谈, 通过定性研究, 判断学习者导致听力焦虑的 主要原因有哪些以及学习者如何降低听力焦虑。主要访谈问题为: 哪些因素 导致听力焦虑？什么时候最容易产生听力焦虑？通常采用什么方法降低听力 焦虑? 为了提高听力效果, 你学会了针对不同的听力任务采用不同的听力策 略吗？听力策略的运用有利于缓解你的焦虑情绪吗？

\section{4. 数据分析}

在正式访谈前, 做了 2 人的先导性研究。为了信息的准确性, 采访采取 了笔记加录音的形式。访谈结束后, 以录音转写的方式进行语料整理, 主要 从三个方面进行：教学因素、个人因素以及遇到听力焦虑时的应对策略。

\section{4. 结果与分析}

在英语听力焦虑因素方面, 本研究的定性分析结果: 非英语专业研究生 
的听力水平、听力材料的特点以及教师讲课模式等是引发听力焦虑的主要根 源。

在 6 名学生的采访中, 感到听力焦虑的因素有: 1) 学习者自身听力水平。 当学习者词汇量过少以及对听力材料背景知识缺少认识时, 都会影响到其听 力理解, 从而产生听力焦虑。2) 听力材料的特点。当听力材料超出调查对象 的水平时, 即会产生焦虑, 因此听力材料的难易程度直接影响听者的焦虑情 绪。3) 听力时间的长短。长时间的听力会导致疲劳, 不够专注, 不能及时调 整自己的状态, 不能保证听力的流利和准确, 增加学习者的心理负担, 因此 会增加听力理解的难度。4) 教学课堂模式。教师在听力课堂的提问和直接纠 错的行为, 会使学习者害怕教师对自己评价不高而导致焦虑。

在降低听力焦虑的应对策略方面，本研究的分析结果：

6 人认为对于学习者本身, 要夯实语言基础, 其中主要包括词汇、句法、 语音等。词汇量的扩大以及辨音能力的提高, 会使听力材料中关键词的抓取 起到至关重要的作用, 同时对于句法的熟练掌握会极大提高听力的理解能力。 5 人认为, 教师应引导学生积极思考, 及时向学生展示对于听力材料 “联想” 和 “预测” 等听力策略的应用, 训练策略可以具体到:通过视频、图片等视觉 辅助, 由个人或者小组形式对听力主要内容进行预测; 提供相关阅读材料, 让 学生熟悉背景知识; 预习并提供原始词汇和解释性概念的词汇表, 使学生听 读结合; 4 人认为教师应充分了解学生的听力水平, 提供合适的听力策略。 在听力材料的选择上, 种类和内容都不能单一, 应由易到难, 循序渐进。

\section{5. 建议}

在英语听力教学中可以从两方面缓解听力焦虑:

第一, 努力改变学生的主观因素, 克服听力焦虑障碍。在语言的基本技 能中, 听力是非常重要的一项, 衡量着学习者语言综合能力的高低。因此, 教师在帮助学生进行听力练习时, 要注意学习者综合能力的培养, 让学生在 学习英语的过程中, 不断完善知识结构和词汇储备。除此以外, 教师应引导 学习者适量加强听力的训练, 增强辨音能力, 为听力能力的提高奠定坚实的 基础。同时, 由于焦虑属于心理学研究范畴, 应用心理学领域的一些具体的 方法, 如针对心理因素引起的焦虑而采用的认知行为疗法 [7]、自我疏导和自 我放松方法等可以帮助缓解焦虑情绪。学生要为不断提高自己的学习能力, 明确自身目标, 端正学习态度, 在教师的积极引导下, 获得更有效的听力策 略和技能, 从而减少听力焦虑。

第二，积极改善教师客观方面的因素，排除听力焦虑。Krashen [4]曾指 出那些可以在低焦虑的环境中提供可理解输入的教学方法是最佳的外语教学 方法。首先，掌握听力技巧有助于对听力材料的理解和把握，有助于降低焦 虑程度, 提高听力理解效果。教师应选择合适的听力材料。二语教师需要明 白每个学生的个体差异, 根据所学生的具体实际水平选择合理有效的教材, 播放听力材料时应采用由易入难、循序渐进的原则; 其次, 帮助学生夯实基 础, 树立听力自信心。外语听者的词汇量是决定其听力理解能力的重要因素。 学生的听力自信来自于扎实的语言基础, 主要包括词汇、句法和辨音。朱艳 
华 [8] 已有研究证实在听力教学中融入元认知策略培训, 能有效降低学生的听 力焦虑感。因此, 教师应在教学中积极引导学生进行听力的训练和及时的自 我纠错, 在不断练习、辨错、修正中逐步提高自己的能力, 并且有意识地减 少焦虑情绪, 进行自我激励。此外, 作为教学评估者, 还应及时改进反馈意 见, 营造轻松和谐的教学氛围, 对问题给予提示, 引导学生正确回答, 对学 生的错误采取宽容积极的态度, 做出积极的评价, 尽量减少可能引起学生焦 虑的教师因素 [9]。

\section{6. 结语}

本研究发现影响非英语专业研究生外语焦虑的三个主要因素在于学习 者、教师和其他因素。结果表明, 外语焦虑主要受学习者因素的影响, 其次 为教师因素和其他因素。其中, 学习者因素受 “学习策略” 因素的影响最大, 教师因素受 “教学方法” 因素的影响最大。对于听力焦虑差异和听力焦虑的 主要因素, 外语教师应从教学实践出发, 提高外语整体水平, 在听力材料的 选择、听力策略的培训和教师的课堂作用上减少学生在听力活动中的焦虑, 提高外语学习效率。本研究的不足在于研究结果还需多次验证, 进一步提高 分析结果的信度和效度。由于笔者学识有限, 时间不够充足及其他一些不可 控制因素, 本研究势必存在一些局限性。本研究的访谈对象选自某大学非英 语专业的研究生。采访不同背景的对象可能会有不同的结果。采访的数量及 英语水平也会影响研究的结果。所以, 本研究缺乏全面的概括性。因此, 未 来研究应该采用不同专业不同大学作样本进行研究以提升研究的效度。同时, 还可以加深对听力焦虑的更深层次的理解。

\section{基金项目}

中北大学第十七届研究生科技立项 “基于 ELAN 的多模态话语研究” (项 目编号：20201751)阶段性成果; 2020 年度山西省哲学社会科学规划课题(编 号 2020YE019)阶段性成果; 山西省教育厅 “基于课程思政理念的“一体两翼 式, 研究生基础英语教学模式探索” 阶段性成果。

\section{Conflicts of Interest}

The authors declare no conflicts of interest.

\section{References}

[1] 张健, 周莉薇. 非英语专业大学生听力焦虑跟踪研究 [J]. 外国语文, 2013(S1): 67-89.

[2] Horwitz, E.K. and Horwitz, M.B.J. (1986) Foreign Language Classroom Anxiety. The Modern Language Journal, 70, 125-128. https://doi.org/10.1111/j.1540-4781.1986.tb05256.x

[3] 李畑英. 外语学习焦虑的心理学和神经生物学分析 [J]. 天津外国语学院学报, 2004, 11(4): 46-51.

[4] Krashen, S.D. (1981) Second Language Acquisition and Second Language Learning. Pergamon Press, Oxford. 
[5] 吕红艳. 非英语专业大学生英语听力焦虑自我图式的发展特点[J]. 淮阴师范学院 学报, 2012, 11(4): 411-414.

[6] 高萌. 外语听力焦虑研究述评[J]. 山东理工大学学报(社会科学版), 2010, 26(6): 162.

[7] 陈永捷. 认知行为疗法通过减轻听力焦虑提高听力成绩的实证研究 [D]: [硕士学 位论文]. 上海: 上海交通大学, 2013.

[8] 朱艳华. 元认知策略训练降低大学生英语听力焦虑的研究 [J]. 湘潭师范学院学 报, 31(5): 220-222.

[9] 周丹丹. 二语课堂中的听力焦虑感和情感策略[J]. 外语教学, 2003(3): 22-29.

\section{Appendix (Abstract and Keywords in Chinese) 非英语专业研究生听力焦虑因素和对应策略的研究}

摘要: 语言焦虑是影响学习效果的重要情绪因素之一, 尤其是在听力活动中。 听力焦虑在英语学习者中普遍存在, 会影响学习者的学习成绩。合理运用听 力学习策略可以减少焦虑对英语学习的负面影响。本文通过访谈的形式对非 英语专业研究生的听力焦虑状况及焦虑原因进行了定性研究, 并有针对性地 提出了克服听力焦虑的对策。

关键词：英语听力，焦虑，对策 